\title{
Publicación científica de los trabajos de grado de maestría de una escuela de posgrado en el Perú
}

\author{
Oscar Mamani-Benito*1; Tomás Caycho-Rodríguez ${ }^{2}$; Madona Tito-Betancur ${ }^{3}$; Josué Turpo Chaparro ${ }^{4}$ \\ ${ }^{1}$ https://orcid.org/0000-0002-9818-2601, Universidad Peruana Unión, Juliaca, Perú, ${ }^{2}$ https://orcid.org/0000-0002-5349-7570, \\ Universidad Privada del Norte, Lima, Perú, ${ }^{3}$ https://orcid.org/0000-0002-4611-6899, Universidad Tecnológica del Perú, Arequipa, \\ Perú, ${ }^{4}$ https://orcid.org/0000-0002-1066-6389, Universidad Peruana Unión, Escuela de Posgrado, Lima, Perú
}

Citar como: Mamani-Benito, O., Caycho-Rodríguez, T., Tito-Betancur, M., \& Turpo Chaparro, J. (2022). Publicación científica de los trabajos de grado de maestría de una escuela de posgrado en el Perú. Revista Digital de Investigación en Docencia Universitaria, 16(1), e1469. https://doi.org/10.19083/ridu.2022.1469

Recibido: 15/04/2021. Revisado: 21/06/2021. Aceptado: 07/10/2021. Publicado: 26/11/2022.

\section{Resumen}

Introducción: Se espera de los trabajos de grado de maestría un aporte al conocimiento científico, por lo tanto, es importante que lleguen a publicarse en revistas científicas indexadas. Objetivo: Determinar la frecuencia de publicación científica de trabajos de grado de maestría de una Escuela de Posgrado en el Perú. Método: Estudio descriptivo de análisis documental en 307 trabajos de grado aprobados en el periodo 2010 - 2019, de ciencias empresariales, ciencias de la salud, psicología, ciencias humanas y educación, ingeniería y arquitectura, salud pública y teología. Resultados: Existe una predominancia de estudios de diseño no experimental (79.5\%) y de tipo correlacional (64.8), además, solo un 11.07\% fueron publicadas en revistas científicas, de las cuales, una está indexada en Scielo y dos en Scopus. Conclusión: La frecuencia de publicación de trabajos de grado de maestría de una Escuela de Postgrado en el Perú, es baja y, aún más su impacto en la comunidad científica internacional.

Palabras clave: publicación periódica; trabajos de grado; estudios de postgrado; Perú.

\section{Scientific publication of master's degree theses of a Peruvian graduate school}

\begin{abstract}
Introduction: Master's theses are expected to contribute to scientific knowledge; therefore, it is important that they are published in indexed scientific journals. Objective: To determine the frequency of scientific publication of master's theses from a graduate school in Peru. Method: Descriptive study of document analysis in 307 theses approved in the period 2010 - 2019, from business sciences, health sciences, psychology, human sciences and education, engineering and architecture, public health and theology. Results: There is a predominance of non-experimental design studies (79.5\%) and correlational type (64.8), in addition, only 11.07\% were published in scientific journals, of which, one is indexed in Scielo and two in Scopus. Conclusion: the frequency of publication of master's theses of a Graduate School in Peru is low, and its impact on the international scientific community is even lower.

Keywords: periodical publication; thesis; graduate studies; Peru.
\end{abstract}




\section{Introducción}

Escribir un trabajo de grado es un requisito esencial para el posgrado y su objetivo es el desarrollo de un espíritu de indagación y exponer al candidato a las técnicas de investigación (Dhaliwal et al., 2010). En tal sentido, un trabajo de posgrado es una actividad planificada que requiere tiempo, por lo que puede realizarse durante varios años y no debe restringirse su divulgación solo a las bibliotecas institucionales o repositorios digitales de trabajos de grado, sino que debe llegar a la comunidad científica a través de publicaciones periódicas (Nour-Eldein et al., 2015).

Es por ello que la publicación de los resultados de un trabajo de grado en una revista revisada por pares es un indicador de su valor científico y aceptabilidad dentro de la comunidad académica (Nieminen et al., 2007). Asimismo, este tipo de publicación conduce a una mejora profesional y satisfacción personal (Ahern, 2012). Sin embargo, en algunos casos la falta de publicación en el grado de maestría se debe a una serie de barreras y factores no-facilitadores que incluyen la motivación del estudiante para publicar, el apoyo y la orientación recibida y otras características como la edad del estudiante, el tipo de trabajo y la carga familiar (Bullen \& Reeve, 2011).

Respecto del tema, la literatura científica da cuenta de investigaciones como la realizada por Tremblay-Wragg et al. (2020) en la que muestra que los estudiantes de maestría y doctorado pueden sentirse aislados mientras escriben sus trabajos de grado, en el periodo posterior a la finalización de los estudios, teniendo únicamente a su asesor para progresar en su trabajo. Asimismo, perciben conductas de intimidación de parte de los científicos superiores hacia los jóvenes estudiantes de posgrado, quizás por la presión que sienten los científicos para competir por clasificaciones diseñadas para medir su valor científico (Mahmoudi et al., 2020). Por otro lado, otro estudio muestra datos interesantes con respecto al género, donde los varones que trabajan con asesoras publican un $10 \%$ más que los estudiantes que trabajan con asesores varones (Pezzoni et al., 2016).

En las últimas décadas, las mayores demandas de calidad y rendición de cuentas, así como la creciente competencia mundial en la clasificación institucional en el sector de la educación superior, han llevado a una presión cada vez mayor sobre la producción científica de posgrado (Cardoso \& Cerecedo, 2019; Mamani \& Apaza, 2019). Como resultado, la publicación en revistas internacionales se ha establecido cada vez más como un requisito explícito de graduación para estudiantes de posgrado, incluso en países no anglófonos ( $\mathrm{Li}$, 2019). Asimismo, la inclusión en los cursos de revisión en las escuelas de posgrado y la inclusión de asignaturas de ética científica muestran la enorme importancia que tiene la finalización del trabajo académico en publicaciones científicas (Rodríguez \& Avello, 2016; Zúñiga, 2019). En este sentido, generar estrategias que permitan a los estudiantes de posgrado tener una mejor comprensión sobre los diferentes procesos de publicación científica y el consecuente aprendizaje de escritura académica es un tema relevante hoy en día en las escuelas de posgrado (Márquez \& Gómez-Zermeño, 2018).

Igual que cualquier investigación, un trabajo de grado no puede considerarse completo si los resultados no están disponibles para las personas que podrían utilizarlos, como la comunidad pública y científica (Sipahi et al., 2012). Por lo tanto, el trabajo de grado debería proporcionar la base para la elaboración de un artículo publicado en una revista de alto impacto. Sin embargo, elaborar un artículo difiere de escribir un trabajo de grado; aun así, aquellos estudiantes que hayan completado un trabajo de grado de maestría o su equivalente deben considerar su publicación (Nour-Eldein et al., 2015; Resta et al., 2010). Al respecto, estudios previos realizados en países desarrollados y en desarrollo advierten que la tasa de publicación de trabajos de grado no es muy alta y varía entre 1.2\% al 52.3\% (Arriola-Quiroz et al., 2010; Dhaliwal et al., 2010; Salbach et al., 2013; Sipahi et al., 2012).

Actualmente se tiene diferentes reportes sobre producción científica peruana en pregrado (De La Cruz-Vargas et al., 2019; Huaraca-Hilario et al., 2017) y de docentes en diferentes áreas (Mamani et al., 2019; Pullido-Medina \& Mejia, 2018); sin embargo, aún son escasos los reportes acerca del 
potencial publicable de los trabajos de grado de maestría en el Perú. Ante este vacío en la literatura, el objetivo de este estudio fue investigar la frecuencia de publicación científica de los trabajos de grado de maestría en revistas internacionales y nacionales. Aunque en la escuela de posgrado donde se hizo el análisis aún no se tiene la política de aceptar trabajos de grado en formato de artículo científico, consideramos que esta investigación es una contribución importante, dado el papel clave que juegan las escuelas de posgrado en el incremento de la productividad científica peruana y que podrían mejorar sus indicadores a mediano plazo.

\section{Método}

\section{Diseño}

Esta investigación corresponde a un estudio descriptivo de análisis de documentos, según la clasificación propuesta por Montero y León (2007).

\section{Unidad de análisis}

Se consideró como unidades de análisis a los trabajos de grado aprobados en el periodo 2010 2019 , con los que egresados de una escuela de posgrado obtuvieron su grado académico de Maestro/ Magíster en una universidad privada del Perú. El universo consta de 319 trabajos de grado de ciencias humanas y educación, teología, salud pública, psicología, ciencias empresariales, ciencias de la salud e ingeniería y arquitectura; cantidad que surgió en función de revisar la accesibilidad total en el repositorio digital de trabajos de grado de la universidad investigada; no obstante, se logró trabajar con 307, pues se excluyeron 12 debido a que no se tenía acceso al contenido completo.

\section{Instrumentos}

Para medir la variable principal, en este caso, la frecuencia de publicación científica de los trabajos de grado de maestría, y variables secundarias, como las características metodológicas, se elaboró una ficha de registro en Excel, la cual estuvo compuesta por ocho ítems en formato lista de cotejo con opciones de respuesta dicotómica (publi- cación científica) y múltiple (tipo de publicación, diseño metodológico, tipo de estudio, tipo de población, tamaño muestral, lugar de ejecución y línea de investigación). Este fue sometido a evaluación por juicio de cuatro expertos (investigadores Renacyt), quienes evaluaron la validez basada en el contenido, a través de su claridad, representatividad y relevancia. El puntaje calculado a través del coeficiente V de Aiken fue de .89, lo cual indica un nivel adecuado (Ventura-León, en prensa).

\section{Procedimientos}

La búsqueda de los trabajos de grado se realizó entre febrero y marzo del 2020, y se utilizó el repositorio digital de la universidad en cuestión. En cada trabajo de grado se evaluó las siguientes variables: unidad de posgrado a la que pertenece, año de aprobación, tipo de investigación, diseño, tipo de población estudiada y tamaño muestral.

Para determinar si los trabajos de grado habían sido publicados en revistas científicas, en primera instancia se hicieron búsquedas digitando el título del trabajo en Google Académico, la base de datos de Scielo y el índice de referencias bibliográficas y citas de Scopus, tanto en español como en inglés. Aunado a esto, como segunda instancia, también se procedió a hacer la búsqueda utilizando los nombres y apellidos del autor(es); para ello se utilizó una estrategia empleada en otro estudio similar (Mejía et al., 2018). Por último, se hizo un rastreo del ID del autor en Google Académico y en ORCID.

Para Google Académico y Scielo:

Autor: Luz Mercedes Mujica Calderón

- "Luz M Mujica».

- «Calderón MMM».

- «Calderón LM».

- "Mujica-Calderón L».

- «Mujica-Calderón LM».

- "Mujica L".

Para Scopus:

- Autor: Luz Mercedes Mujica Calderón

- Apellidos del autor: Mujica Calderón

- Primer nombre del autor: L.M.

Esta investigación fue autofinanciada. Por la naturaleza y ubicación de los datos no fue necesario el consentimiento informado ni alguna autorización del comité de ética, puesto que los trabajos 
de grado analizados son de carácter público, no obstante, se guardó confidencialidad de los autores y asesores involucrados.

\section{Análisis de datos}

Los datos que fueron recolectados se tabularon en el programa Excel 2016. Se hizo un análisis descriptivo y se presentaron los resultados en tablas de frecuencia y porcentaje absolutos.

\section{Resultados}

Se evaluaron 307 trabajos de grado de maestría. En mayor proporción se observan trabajos de grado de la unidad de Ciencias humanas y Educación (35.8\%), seguido por Psicología (14.7\%), Salud Pública (14.7\%) y Teología (14.7), y en menor proporción aparecen Ciencias empresariales (11.7\%), Ciencias de la salud (5.2\%) e Ingeniería y Arquitectura (3.3\%). En cuanto al año de aprobación, se observa un mayor porcentaje de trabajos de grado aprobados en el 2018 (26.1\%), seguido de 2017 (21.2\%), 2014 (18.2\%), 2015 (16.6\%) y 2016 (15.6\%), y en menor porcentaje en los años 2011, 2012 y 2013 (Ver Tabla 1).

En cuanto al tipo y diseño de investigación, el $79.5 \%$ son de diseño no experimental, $18.6 \%$ experimental y $2 \%$ mixto. En la misma línea, en mayor proporción se observa un $64.8 \%$ de estudios correlacionales, y en menor proporción estudios explicativos (12.4\%), estudios que implican la aplicación de un programa de intervención (8.4\%), estudios teóricos (3.6\%), descriptivos (3.3\%), tecnológicos de carácter aplicado (2.9\%), mientras que menos del $2 \%$ fueron estudios de caso, exploratorios, históricos e instrumentales (Ver Tabla 1).

Respecto del tipo de población y tamaño muestral, el mayor porcentaje de trabajos de investigación tuvo participantes de escuelas y colegios (36.2\%), seguido por universitarios (24.8\%), y población eclesiástica (15.6\%); mientras que en menor proporción se trabajó con empresas (7.2\%) e instituciones del estado (7.8\%). Finalmente, la mayoría de estudios contó con la participación de 101 a 200 personas (24.4\%), menos de 50 (21.8\%), 51 a 100 (20.5\%) y 201 a 300 (18.6\%); y en menor porcentaje existen estudios que tuvieron una muestra entre
401 a 600 participantes (9.1\%) y más de 601 (5.5\%).

La Tabla 2 muestra los reportes de publicación científica de trabajos de grado, según unidad de posgrado y año de divulgación. Se observa que entre el periodo 2010-2014 no se difundió ningún resultado de trabajos de grado en alguna revista científica. En cambio, se observa que en el 2017 se logró la mayor frecuencia de publicación (13), luego en el 2019 (6) y en el 2016 (6); mientras que la frecuencia es menor en los años 2015 (4) y 2018 (2). Por último, la unidad con mayor cantidad de trabajos de grado publicados es la de Ciencias empresariales (14), seguida por Ciencias humanas y Educación (9), Ciencias de la salud (5), Teología (3), Psicología (2), Salud Pública (1) e Ingeniería y Arquitectura con ninguna.

A continuación, la Figura 1 muestra la frecuencia de publicación de trabajos de grado de una escuela de posgrado peruana, según año de divulgación. De 307 trabajos de grado, se logró publicar 34 (11.07\%). La mayor tasa de publicación se encuentra en el año 2017 (13), seguido por el 2019(9), y en menor frecuencia en los años 2016 (6); 2015 (4) y 2018 solo dos.

En la tabla 3 se reportan las características metodológicas de los trabajos de grado que fueron publicados en revistas científicas. En este caso, 33 fueron publicados como artículos científicos originales y uno como artículo teórico, aclarando que en todos los casos estas publicaciones representan los resultados expuestos en los trabajos de grado.

En ese sentido, en mayor proporción, los trabajos publicados obedecen a un diseño no experimental (91.2\%), de tipo correlacional (79.4\%), tomando como población a universitarios (23.5\%), seguido de docentes (11.8\%), estudiantes de educación básica regular (11.8\%) y personal de salud como enfermeras (11.8\%). El tamaño muestral más frecuente fue aquel que involucró entre 51 a 200 participantes (41.2\%). Por otro lado, en cuanto al lugar de ejecución, en mayor proporción fueron realizados en provincias y departamentos del Perú (44.1\%), en cambio en Lima capital un 38.2\%, y en el extranjero 17.6\%. Finalmente, la línea de investigación más frecuente corresponde a Liderazgo y Gestión Educativa (23.5\%), seguido por Psicología Organizacional (20.6\%) y Factores Psicológicos del Entorno Educativo (14.7\%). 
Tabla 1

Características de los trabajos de grado de maestría de una escuela de posgrado en el Perú

\begin{tabular}{|c|c|c|c|}
\hline Variable & Categoría & $f$ & $\%$ \\
\hline & Ciencias empresariales & 36 & 11.7 \\
\hline & Ciencias de la salud & 16 & 5.2 \\
\hline & Psicología & 45 & 14.7 \\
\hline \multirow[t]{8}{*}{ Unidad de posgrado } & Ciencias humanas y Educación & 110 & 35.8 \\
\hline & Ingeniería y Arquitectura & 10 & 3.3 \\
\hline & Salud pública & 45 & 14.7 \\
\hline & Teología & 45 & 14.7 \\
\hline & 2011 & 1 & .3 \\
\hline & 2012 & 2 & .7 \\
\hline & 2013 & 4 & 1.3 \\
\hline & 2014 & 56 & 18.2 \\
\hline \multirow[t]{10}{*}{ Año de aprobación } & 2015 & 51 & 16.6 \\
\hline & 2016 & 48 & 15.6 \\
\hline & 2017 & 65 & 21.2 \\
\hline & 2018 & 53 & 17.2 \\
\hline & 2019 & 27 & 8.7 \\
\hline & Descriptivo & 10 & 3.3 \\
\hline & Correlacional & 199 & 64.8 \\
\hline & Explicativo & 38 & 12.4 \\
\hline & Estudio de caso & 4 & 1.3 \\
\hline & Programa de intervención & 25 & 8.1 \\
\hline \multirow[t]{6}{*}{ Tipo de estudio } & Exploratorio & 2 & .7 \\
\hline & Teórico & 11 & 3.6 \\
\hline & Histórico & 5 & 1.6 \\
\hline & Instrumental & 4 & 1.3 \\
\hline & Tecnológica/aplicada & 9 & 2.9 \\
\hline & Experimental & 57 & 18.6 \\
\hline \multirow[t]{5}{*}{ Diseño } & No experimental & 244 & 79.5 \\
\hline & Mixto & 6 & 2.0 \\
\hline & Empresa & 22 & 7.2 \\
\hline & Comunidad & 17 & 5.5 \\
\hline & Escuela y colegio & 111 & 36.2 \\
\hline \multirow[t]{7}{*}{ Tipo de población } & Universidad & 76 & 24.8 \\
\hline & Instituciones del Estado & 24 & 7.8 \\
\hline & Eclesiástico & 48 & 15.6 \\
\hline & Otros & 9 & 2.9 \\
\hline & Menos de 50 & 67 & 21.8 \\
\hline & Entre 51 a 100 & 63 & 20.5 \\
\hline & Entre 101 a 200 & 75 & 24.4 \\
\hline \multirow[t]{3}{*}{ Tamaño muestral } & Entre 201 a 400 & 57 & 18.6 \\
\hline & Entre 401 a 600 & 28 & 9.1 \\
\hline & Más de 600 & 17 & 5.5 \\
\hline
\end{tabular}




\section{Tabla 2}

Frecuencia de publicación según unidades de posgrado: periodo 2010 - 2019

\begin{tabular}{|c|c|c|c|c|c|c|c|}
\hline \multirow{2}{*}{ Unidad } & \multicolumn{6}{|c|}{ Año de publicación } & \multirow{2}{*}{ Sub Total } \\
\hline & $2010-2014$ & 2015 & 2016 & 2017 & 2018 & 2019 & \\
\hline Ciencias empresariales & - & 1 & 2 & 6 & 2 & 3 & 14 \\
\hline Ciencias de la salud & - & 2 & 1 & 1 & - & 1 & 5 \\
\hline Psicología & - & - & 1 & 1 & - & - & 2 \\
\hline Ciencias humanas y Educación & - & 1 & 1 & 3 & - & 4 & 9 \\
\hline Ingeniería y Arquitectura & - & - & - & - & - & - & - \\
\hline Salud pública & - & - & - & 1 & - & - & 1 \\
\hline Teología & - & - & 1 & 1 & - & 1 & 3 \\
\hline Ciencias empresariales & - & 4 & 6 & 13 & 2 & 9 & 34 \\
\hline
\end{tabular}

\section{Figura 1}

Frecuencia de publicación de los trabajos de grado según año de divulgación

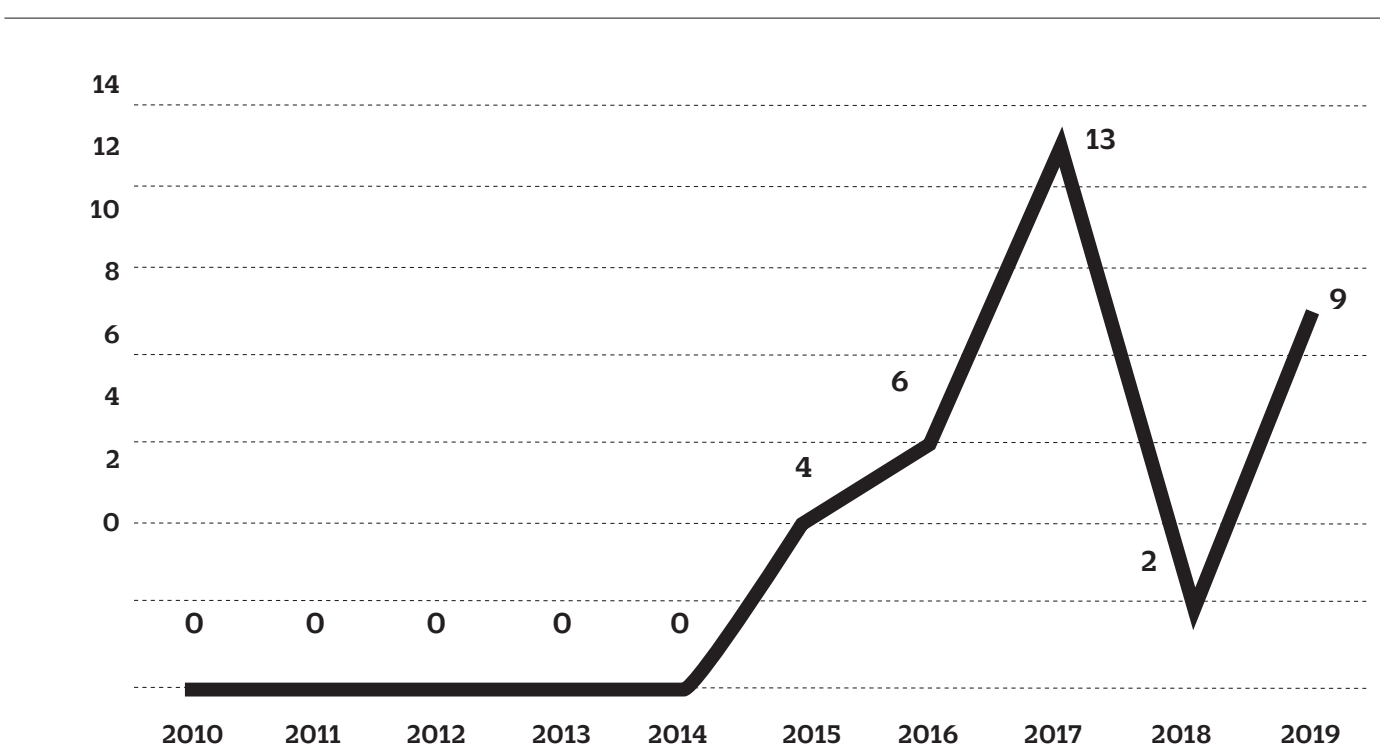

En la Tabla 4 se muestran las características de las revistas donde se publicaron los trabajos de grado de maestría. En este caso, siete son revistas institucionales y tres científicas; así, se observa mayor cantidad de publicaciones en la revista Muro de la investigación, Valor agregado y Revista científica de ciencias de la salud, todas del ámbito nacional (Revistas institucionales). En cambio, en revistas indexadas en Scopus y Scielo, como es el caso de Espacios (discontinuado en Scopus), Revista de investigación en ciencias sociales y la Revista de Psicología (Revistas científicas), solo hay cuatro publicaciones. Por último, hay tres revistas (Muro de la Investigación, Valor Agregado y Valor Contable) que aún no se encuentran indexadas. 
Tabla 3

Características de los trabajos de grado publicados en revistas científicas $(n=34)$

\begin{tabular}{|c|c|c|c|}
\hline Variable & Categoría & $f$ & $\%$ \\
\hline \multirow[t]{2}{*}{ Publicado como } & Articulo original & 33 & 97.06 \\
\hline & Articulo teórico & 1 & 2.94 \\
\hline \multirow[t]{3}{*}{ Diseño } & No experimental & 31 & 91.2 \\
\hline & Preexperimental o cuasi experimental & 3 & 8.8 \\
\hline & Correlacional & 27 & 79.4 \\
\hline \multirow[t]{6}{*}{ Tipo } & Explicativo & 6 & 17.6 \\
\hline & Teórico & 1 & 2.9 \\
\hline & Universitarios & 8 & 23.5 \\
\hline & Ejecutivos y administradores & 3 & 8.8 \\
\hline & Docentes & 4 & 11.8 \\
\hline & Estudiantes de Educación Básica & 4 & 11.8 \\
\hline \multirow[t]{9}{*}{ Tipo de población } & Enfermeras & 4 & 11.8 \\
\hline & Indígenas & 1 & 2.9 \\
\hline & Líderes religiosos & 2 & 5.9 \\
\hline & Feligreses & 3 & 8.8 \\
\hline & Colaboradores de empresa & 2 & 5.9 \\
\hline & Pacientes ambulatorios & 1 & 2.9 \\
\hline & Documentos & 2 & 5.9 \\
\hline & Menos de 50 participantes & 9 & 26.5 \\
\hline & Entre 51 a 200 & 14 & 41.2 \\
\hline \multirow[t]{4}{*}{ Tamaño muestral } & Entre 201 a 600 & 10 & 29.4 \\
\hline & Más de 600 & 1 & 2.9 \\
\hline & Lima, Perú & 13 & 38.2 \\
\hline & Provincia, Perú & 15 & 44.1 \\
\hline \multirow[t]{6}{*}{ Lugar de ejecución } & Extranjero & 6 & 17.6 \\
\hline & Total & 34 & 100.0 \\
\hline & Psicología organizacional & 7 & 20.6 \\
\hline & Liderazgo y gestión educativa & 8 & 23.5 \\
\hline & Contabilidad financiera & 2 & 5.9 \\
\hline & Auditoría & 1 & 2.9 \\
\hline \multirow[t]{7}{*}{ Línea de investigación } & Psicología clínica y de la salud & 3 & 8.8 \\
\hline & Psicología positiva & 3 & 8.8 \\
\hline & Factores psicológicos en el entorno educativo & 5 & 14.7 \\
\hline & Enfermería en administración y gestión & 1 & 2.9 \\
\hline & Salud pública & 1 & 2.9 \\
\hline & Investigación bíblico-aplicada & 2 & 5.9 \\
\hline & Investigación bíblico-histórica & 1 & 2.9 \\
\hline
\end{tabular}


Tabla 4

Características de las revistas científicas

\begin{tabular}{lllll}
\hline Revista de publicación & País & Tipo de revista & Indexación & Cantidad \\
\hline & & & & 9 \\
Revista Muro de la Investigación & Perú & Revista institucional & No & 8 \\
Revista de Investigación Valor Agregado & Perú & Revista institucional & No & 6 \\
Revista Científica de Ciencias de la Salud & Perú & Revista institucional & Latindex & 3 \\
Revista de Investigación Universitaria & Perú & Revista institucional & Latindex & 2 \\
Revista de Investigación Valor Contable & Perú & Revista institucional & No & 2 \\
Revista Espacios & Venezuela & Revista científica & Scopus & 1 \\
Investigación en Ciencias Sociales & Paraguay & Revista científica & Scielo & 1 \\
Revista de Psicología (PUCP) & Perú & Revista científica & Scopus & 1 \\
Estrategias para el Cumplimiento de la Misión & Perú & Revista institucional & Latindex \\
Revista Apuntes Universitarios & Perú & Revista institucional & WoS (ESCI) & 1 \\
\hline
\end{tabular}

\section{Discusión}

El objetivo para realizar un trabajo de grado de maestría es formar al candidato a magister en métodos de investigación y en el desarrollo del pensamiento científico, por lo que los resultados obtenidos merecen ser difundidos (Dhaliwal et al., 2010).

En tal sentido, se ha sugerido que el valor real de un trabajo científico radica en su publicación en una revista indexada, donde se hacen visibles y fácilmente accesibles los resultados de la investigación en cualquier parte del mundo (Dhaliwal \& Kumar, 2008; Nieminen et al., 2007). Además, permite mejorar la credibilidad académica y profesional de los investigadores y de la institución a la cual pertenecen (Abdulmajeed et al., 2014), no obstante, a pesar de su importancia, muchos trabajos de grado permanecen inéditos. Ante ello, el presente estudio tuvo como objetivo investigar la frecuencia de publicación científica de los trabajos de grado de maestría de una universidad peruana en revistas internacionales y nacionales; que, hasta donde se sabe, es uno de los primeros acercamientos empíricos al fenómeno de la publicación de productos científicos de maestría en el Perú.

Los hallazgos sugieren que la tasa de publicación de trabajos de grado de maestría es baja e inferior al reportado por otras investigaciones que varían entre el 30\% y 50\% (Arriola-Quiroz et al., 2010; Caan \& Cole, 2012; Dhaliwal et al., 2010; Frković et al., 2003; Salbach et al., 2013). Sin embargo, el bajo porcentaje de trabajos publicados basados en los trabajos de grado no es una característica exclusiva del Perú. Por ejemplo, Yaman y Atay (2007) analizaron 227 trabajos de grado turcos de ciencias del deporte e informaron una tasa de publicación del $1.7 \%$ en revistas internacionales. Asimismo, en Francia, solo el 17\% de los trabajos de grado de doctores en Medicina dan como resultado la publicación de un artículo (Salmi et al., 2001). Finalmente, en Croacia, solo entre el $13 \%$ y $14 \%$ de trabajos de grado resultaron en artículos publicados (Frkovic et al., 2003).

Se reportó también que las tasas de publicación de trabajos de grado en revistas internacionales eran aproximadamente el $8 \%$ de las tasas de publicación en revistas nacionales. Esto puede explicarse posiblemente porque los temas de los trabajos de grado podrían ser encontrados como problemas locales por revisores de revistas internacionales.

Otro hallazgo importante tiene que ver con algunas características metodológicas predominantes en los trabajos de grado de maestría, pues en mayor proporción corresponden a diseños no experimentales y de tipo correlacional. Este hecho difiere con lo hallado por Rosales et al. (2010) 
quienes, al analizar trabajos de grado de Maestría en salud en Cuba, encontraron mayor proporción de estudios experimentales, al igual que Pacheco et al. (2018) en Venezuela, quienes analizaron trabajos de grado de maestría en Educación Matemática, hallando predominancia de estudios cuasi experimentales. En cambio, es similar a lo hallado en los trabajos de pregrado, tal como lo demuestra un estudio en Medicina (Castro-Maldonado et al., 2015) y otro en Psicología (Mamani, 2018).

Con relación al hallazgo anterior, una explicación ante el hecho de que se elijan plantear trabajos para el grado de maestría bajo un diseño de investigación básico, podría involucrar algunos factores como el de querer obtener el grado académico a la brevedad, evitando evaluaciones engorrosas por parte del jurado dictaminador y el director de trabajos de grado (Ochoa \& Moreno, 2019). Otros factores serían no complicarse a la hora de hacer la estadística (Cardoso \& Cerecedo, 2019), deficiencias en metodología de la investigación (Reyes-Cruz \& Gutiérrez, 2015) y poco involucramiento del asesor (Hernández et al., 2016).

En relación con lo anterior, llama la atención que similares características también son notorias en los trabajos de grado que alcanzaron ser publicados en revistas científicas; no obstante, se sabe que el diseño y tipo de investigación no son los únicos criterios relevantes a la hora de evaluar los manuscritos científicos, pues el comité editorial o los pares revisores suelen darle mayor importancia al lenguaje científico, la importancia del tema, la relevancia de los resultados, entre otros aspectos (Rivas, 2017). En tal sentido, la calidad de las investigaciones sería otro motivo de investigación para futuros estudios, pues a juzgar por la calidad de las revistas donde fueron publicados los trabajos de grado de maestría, pocos se encuentran indexados en Scielo y Scopus.

Al interpretar los resultados del estudio, se deben considerar algunas limitaciones. Primero, los resultados no son automáticamente comparables con los de otros programas de posgrado, porque solo se han analizado los trabajos de grado de una sola universidad; además, el énfasis en la investigación y publicación puede variar entre los programas de maestría. Segundo, es posible que no se pudo acceder a algunos artículos derivados de trabajos de grado debido al hecho de que sus títulos pudieran haber cambiado en función de las recomendaciones de los revisores de las revistas donde fueron publicados. Tercero, la información sobre la publicación de los trabajos de grado se actualizó hasta marzo de 2020, por lo que es posible que se publicaran más trabajos de grado después de esa fecha. A pesar del alcance limitado del estudio los hallazgos pueden ser valiosos para otros programas de maestría, especialmente del Perú, que consideren plantear intervenciones en la formación de posgrado. Por lo tanto, otros estudios podrían evaluar la fortaleza de los resultados en otros programas de maestría que permitan la comparación entre ellos.

Se concluye que la tasa de publicación de trabajos de grado de maestría es baja y se realiza principalmente en revistas que no están indexadas en bases de datos internacionales. Ante esta situación, se deben generar soluciones para promover la publicación de trabajos de grado de posgrado. Aunque la escuela de posgrado de la universidad donde se hizo la investigación aún no tiene la política de aceptar artículos en vez de trabajos de grado, para obtener el grado académico, la obligación de preparar uno o más manuscritos listos para ser enviados a una revista o colocar las publicaciones científicas de trabajos de grado como uno de los criterios para las promociones o ascensos académicos pueden ser alternativas adecuadas para incrementar las tasas de publicación (Sipahi et al., 2012). Por otra parte, quizás el respaldo institucional, el reconocimiento y la recompensa por el trabajo publicado puedan ayudar a incrementar la tasa reportada. Sin embargo, estas propuestas pueden resultar insuficientes para aumentar las tasas de publicación (Salmi et al., 2001).

\section{Referencias}

Abdulmajeed, A. A., Ismail, M. A., \& Nour-Eldein, H. (2014). Research publications in medical journals (19922013) by family medicine authors-Suez Canal University-Egypt. Journal of Family Medicine and Primary Care, 3(4), 368-373. https://pubmed.ncbi.nlm. nih.gov/25657945/

Ahern, K. (2012). How to create a journal article from a the- 
sis. Nurse researcher, 19(4), 21-25. https://journals. rcni.com/doi/abs/10.7748/nr2012.07.19.4.21.c9220

Arriola-Quiroz, I., Curioso, W. H., Cruz-Encarnacion, M., \& Gayoso, O. (2010). Characteristics and publication patterns of theses from a Peruvian medical school. Health Information \& Libraries Journal, 27(2), 148154. https://doi.org/10.1111/j.1471-1842.2010.00878.x

Bullen, C. R., \& Reeve, J. (2011). Turning postgraduate students' research into publications: A survey of New Zealand masters in public health students. Asia Pacific Journal of Public Health, 23(5), 801-809. https://doi.org/10.1177/1010539511417998

Caan, W., \& Cole, M. (2012). How much doctoral research on clinical topics is published? BMJ Evidence-Based Medicine, 17(3), 71-74. https://doi.org/10.1136/ebmed-2011-100227

Cardoso, E. O., \& Cerecedo, M. T. (2019). Valoración de las Competencias Investigativas de los Estudiantes de Posgrado en Administración. Formación Universitaria, 12(1), 35-44. https://doi.org/10.4067/s071850062019000100035

Castro-Maldonado, B., Callirgos-Lozada, C.C., Caicedo-Písfil, M.K., Plasencia-Dueñas, E. A., \& Díaz-Vélez, C. (2015). Características de los trabajos de grado de pregrado de Medicina de una universidad pública del Perú. Horizonte Médico, 15(3), 34-39. http:// www.scielo.org.pe/scielo.php?script=sci_arttext\&pid=S1727-558X2015000300006

De La Cruz-Vargas, J. A., Correa-Lopez, L. E., Alatrista-Gutierrez de Bambaren, M.D.S., Sanchez, H. H., Luna, C. Loo, M., Cano, L., Gonzalez, M., Salinas, C., Segura, P., Alva, M., Morales, E., Castillo, E., Oshiro, S., Machado, A., Sanchez, D., Chanduvi, W., \& Roldan, L. (2019). Promoting research in medical students and increasing scientific production in universities: Experience of the Undergraduate Thesis Workshop Course. Educacion Medica, 20(4), 199-205. https:// doi.org/10.1016/j.edumed.2018.06.003

Dhaliwal, U., \& Kumar, R. (2008). An observational study of the proceedings of the All India Ophthalmological Conference, 2000 and subsequent publication in indexed journals. Indian Journal of Ophthalmology, 56(3), 189-195. https://www.ncbi.nlm.nih.gov/ pmc/articles/PMC2636100/

Dhaliwal, U., Singh, N., \& Bhatia, A. (2010). Masters theses from a university medical college: Publication in indexed scientific journals. Indian Journal of Ophthalmology, 58(2), 101-104. https://doi. org/10.4103/0301-4738.60070

Frković, V., Skender, T., Dojcinovic, B., \& Bilic-Zulle, L. (2003). Publishing scientific papers based on Master's and $\mathrm{Ph}$. D. theses from a small scientific community: case study of Croatian medical schools. Croatian Medical Journal, 44(1), 107-111. https://pubmed. ncbi.nlm.nih.gov/12590439/

Hernández, C.A., Jiménez, M., Guadarrama, E., \& Rivera, A.E. (2016). La Percepción de la Motivación y Satisfac- ción de la Tutoría Recibida en Estudios de Posgrado. Formación Universitaria, 9(2), 49-58. https://doi. org/10.4067/S0718-50062016000200006

Huaraca-Hilario, C. M., Apaza, A., \& Mejía, C. (2017). Realidad peruana de la publicación científica estudiantil en los últimos diez años. Educacion Médica Superior, 31(3), 124-134. http://www.ems.sld.cu/index.php/ ems/article/view/1019

Li, Y. (2019). Mentoring Junior Scientists for Research Publication. In P. Habibie \& K. Hyland (Eds.), Novice Writers and Scholarly Publication: Authors, Mentors, Gatekeepers (pp. 1-297). http://dx.doi. org/10.1007/978-3-319-95333-5_13

Mahmoudi, M., Ameli, S., \& Moss, S. (2020). The urgent need for modification of scientific ranking indexes to facilitate scientific progress and diminish academic bullying. BioImpacts, 10(1), 5-7. https://doi. org/10.15171/bi.2019.30

Mamani, O. J., \& Apaza, E. E. (2019). Rasgo conciencia y actitud hacia la trabajos de grado en universitarios de una sociedad científica. Revista de Psicología, 37(2), 559-581. https://doi.org/10.18800/psico.201902.008

Mamani, O.J, Ventura-León, J., \& Caycho, T. (2019). Publicación científica de docentes que conforman el jurado de trabajos de grado en una Facultad de Ciencias de la Salud peruana. Revista Cubana de Información en Ciencias de la Salud, 30(3), 1-9. http://scielo.sld.cu/scielo.php?script=sci_arttext\&pid=S2307-21132019000300008\&lng=es\&nrm=iso\&tlng=es

Mamani, O.J. (2018). Calidad metodológica y características de los trabajos de grado de pregrado de psicología de una universidad privada del Perú. Propósitos y representaciones, 6(2), 301-319. https://doi. org/10.20511/pyr2018.v6n2.224

Márquez, S., \& Gómez-Zermeño, M. G. (2018). Grupo Virtual de escritura académica: Una e-innovación para impulsar la publicación científica. Revista Mexicana de Investigacion Educativa, 23(76), 203-227. http://


t\&pid=S1405-66662018000100203

Mejia, C. R., Valladares-Garrido, M. J., \& Valladares-Garrido, D. (2018). Baja publicación en revistas científicas de médicos peruanos con doctorado o maestría: Frecuencia y características asociadas. Educacion Medica, 19, 135-141. https://doi.org/10.1016/j.edumed.2017.01.009

Montero, I., \& León, O. G. (2007). A guide for naming research studies in Psychology. International Journal of Clinical and Health Psychology, 7(3), 847-862. https://www.redalyc.org/pdf/337/33770318.pdf

Nieminen, P., Sipilä, K., Takkinen, H. M., Renko, M., \& Risteli, L. (2007). Medical theses as part of the scientific training in basic medical and dental education: experiences from Finland. BMC Education, 7(1), 51. https://doi.org/10.1186/1472-6920-7-51

Nour-Eldein, H., Mansour, N., \& Abdulmajeed, A. (2015). 
Master's and doctoral theses in family medicine and their publication output, Suez Canal University, Egypt. Journal of Family Medicine and Primary Care, 4(2), 162-167. https://pubmed.ncbi.nlm.nih. gov/25949959/

Ochoa, L., \& Moreno, E. (2019). Análisis de comentarios escritos de directores de trabajos de grado de posgrado. Revista Colombiana de Educación, 76, 143-171. http://www.scielo.org.co/pdf/rcde/n76/0120-3916rcde-76-143.pdf

Pacheco, V., Martínez-Padrón, O.J., \& Enrique, F.E. (2018). Análisis de los Trabajos de Grado de la Maestría en Educación Matemática de la Universidad de Carabobo: 2005-2014. Unión, 14(53), 159-180. https://union.fespm.es/index.php/UNION/issue/ view/61/59

Pezzoni, M., Mairesse, J., Stephan, P., \& Lane, J. (2016). Gender and the publication output of graduate students: A case study. PLOS ONE, 11(1), 1-12. https:// doi.org/10.1371/journal.pone.0145146

Pulido-Medina, C., \& Mejia, C.R. (2018). Publicación científica de los docentes de medicina en una universidad colombiana: características y factores asociados. Revista Médica Superior, 32(2), 1-9. http://ems.sld. cu/index.php/ems/article/view/1294/670

Resta, R. G., McCarthy-Veach, P. M., Charles, S., Vogel, K., Blase, T., \& Palmer, C. G. (2010). Publishing a Master's thesis: A guide for novice authors. Journal of Genetic Counseling, 19(3), 217-227. https://doi.org/10.1007/ s10897-009-9276-2

Reyes-Cruz, M.R., \& Gutiérrez, J.M. (2015). Sentido de autoeficacia en investigación de estudiantes de posgrado. Sinéctica, 45, 1-15. http://www. scielo.org. $\mathrm{mx} / \mathrm{scielo}$.php?script=sci_arttext \&pi$\mathrm{d}=$ S1665-109X2015000200011

Rodríguez, R., \& Avello, R. (2016). La revisión por pares como evaluación formativa en un curso de posgrado sobre publicación científica. Educacion Médica Superior, 30(4), 361-371. http://scielo.sld.cu/scielo.php?script=sci_arttext\&pid=S0864-21412016000400012

Rosales, B., Machado, M.J., Garcia, A.B., \& Alegrant, M.L. (2010). Análisis bibliométrico de los trabajos de grado de maestría sobre salud de la provincia Ciego de Ávila (2007-2009). Mediciego, 16(2), 0. http:// www.revmediciego.sld.cu/index.php/mediciego/ article/view/1197/1287

Rivas, F. (2017). Cómo publicar un artículo original en revistas científicas con factor de impacto. Pediatría Atención Primaria, 19(26), 101-109. http:// scielo.isciii.es/scielo.php?script=sci_arttext\&pi$d=$ S1139-76322017000300014

Salbach, N. M., O’Brien, K., Evans, C., \& Yoshida, K. (2013). Dissemination of student research in a Canadian Master of Science in Physical Therapy programme. Physiotherapy Canada, 65(2), 154-157. https://doi. org/10.3138/ptc.2012-18

Salmi, L. R., Gana, S., \& Mouillet, E. (2001). Publication pattern of medical theses, France, 1993-98. Medical Education, 35(1), 18-21. https://doi.org/10.1111/j.13652923.2001.00768.x

Sipahi, H., Durusoy, R., Ergin, I., Hassoy, H., Davas, A., \& Karababa, A. O. (2012). Publication rates of public health theses in international and national peer-review journals in Turkey. Iranian Journal of Public Health, 41(9), 31-35. https://pubmed.ncbi.nlm.nih. gov/23193503/

Tremblay-Wragg, E., Mathieu, S., Labonté-Lemoyne, E., Déri, C.E., \& Gadbois, M. E. (2020). Writing more, better, together: how writing retreats support graduate students through their journey. Journal of Further and Higher Education, 45(1), 95-106. https://doi.org/10.1080/0309877X.2020.1736272

Ventura-León, J. (en prensa). De regreso a la validez basada en el contenido. Adicciones. https://www.adicciones.es/index.php/adicciones/article/view/1213/1038

Yaman, H., \& Atay, E. (2007). PhD theses in Turkish sports sciences: A study covering the years 1988-2002. Scientometrics, 71, 415-421. https://doi.org/10.1007/ s11192-007-1679-y

Zúñiga, J. P. (2019). Comportamiento ético en la publicación científica: malas conductas y acciones para evitarlas. Revista Educación, 44(1). https://doi. org/10.15517/revedu.v44i1.35548

RIDU / Revista Digital de Investigación en Docencia Universitaria / e-ISSN: 2223-2516

(c) Los autores. Este artículo es publicado por la Revista Digital de Investigación en Docencia Universitaria del Área de Institutional Research and Effectiveness de la Dirección de Aseguramiento de la Calidad, Universidad Peruana de Ciencias Aplicadas. . "Este es un artículo de acceso abierto, distribuido bajo los términos de la LicenciaCreativeCommons Atribución 4.0 Internacional (CC BY 4.0) (https://creativecommons.org/licenses/by/4.0/deed.es), que permite el uso, distribución y reproducción en cualquier medio, siempre que la obra original sea debidamente citada." 